Die mittlere Nachbeobachtungszeit betrug 59 Monate.

Im Ergebnis zeigte sich eine Reduktion der Restenosierung durch DES von $16,5 \%$ gegenüber $19,8 \%$ in der Gruppe, die einen BMS erhalten hatte (Hazard Ratio 0,76). Auch die Rate der definitiven Stentthrombosen war mit $0,8 \%$ gegenüber $1,2 \%$ niedriger. Im primären Endpunkt, dem Eintreten von Tod jeglicher Ursache oder nicht tödlichem Herzinfarkt, gab es zwischen beiden Behandlungsgruppen keinen Unterschied. Auch hinsichtlich der Lebensqualität gab es keinen Unterschied zwischen den beiden Behandlungsgruppen.
Bønaa KH et al. Drug-Eluting or Bare-Metal Stents for Coronary Artery Disease. N Engl J Med. 2016:375:1242-1252

\section{Kommentar}

Die Ergebnisse der NORSTENT-Studie sind bei der ersten Präsentation während des ESC-Kongresses mit Raunen aufgenommen worden. Dabei bestätigen sie genau das, was von medikamentenbeschichteten Stents a priori erwartet wurde: eine Reduktion der Restenoserate. Weitergehende Therapie- ziele wie eine Reduktion der Sterblichkeit oder nicht schwerwiegender Myokardinfarkte oder gar eine Verbesserung der Lebensqualität konnten in der
NORSTENT-Studie jedoch nicht erzielt werden. Somit bleiben die medikamentenbeschichteten Stents weiterhin Goldstandard in der interventionellen Koronartherapie. Bei bestimmten Risikokollektiven (z. B. Indikation zur effektiven Antikoagulation) kann jedoch ohne schlechtes Gewissen auf einen Metallstent zurückgegriffen werden.

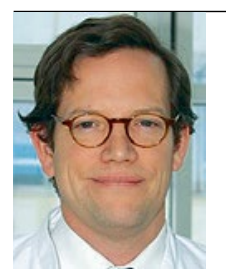

Prof. Dr. med. Peter W. Radke Klinik für Innere Medizin Kardiologie, Schön Klinik Neustadt

\title{
Wie genau ist die Blutdruckmessung am Handgelenk?
}

\section{Die Blutdruckmessung am Handgelenk erfreut sich seit Jahren großer Beliebt-}

heit. Über die Präzision dieser Messung unter Alltagsbedingungen in der Allgemeinbevölkerung liegen bisher relativ wenig detaillierte Informationen vor.

$\mathrm{D}$ ie vorliegende Arbeit untersuchte die Genauigkeit der Handgelenksmessung an 721 unselektierten Probanden der Allgemeinbevölkerung, die selbst den Blutdruck am Handgelenk gemessen haben. Nach einer Einweisung wurde der Blutdruck sowohl in der Sprechstunde als auch zuhause sowohl am Oberarm als auch am Handgelenk gemessen. Die Differenz zwischen der Mes- sung am Oberarm und am Handgelenk wurde sowohl in der Sprechstunde als auch zuhause erhoben. Der Unterschied zwischen der Oberarm-HandgelenksDifferenz zuhause und in der Sprechstunde wurde als Messfehler definiert.

In der Sprechstunde war der systolische Blutdruck am Handgelenk 2,5\% niedriger als am Oberarm $(p=0,002)$, während zuhause der Blutdruck sowohl

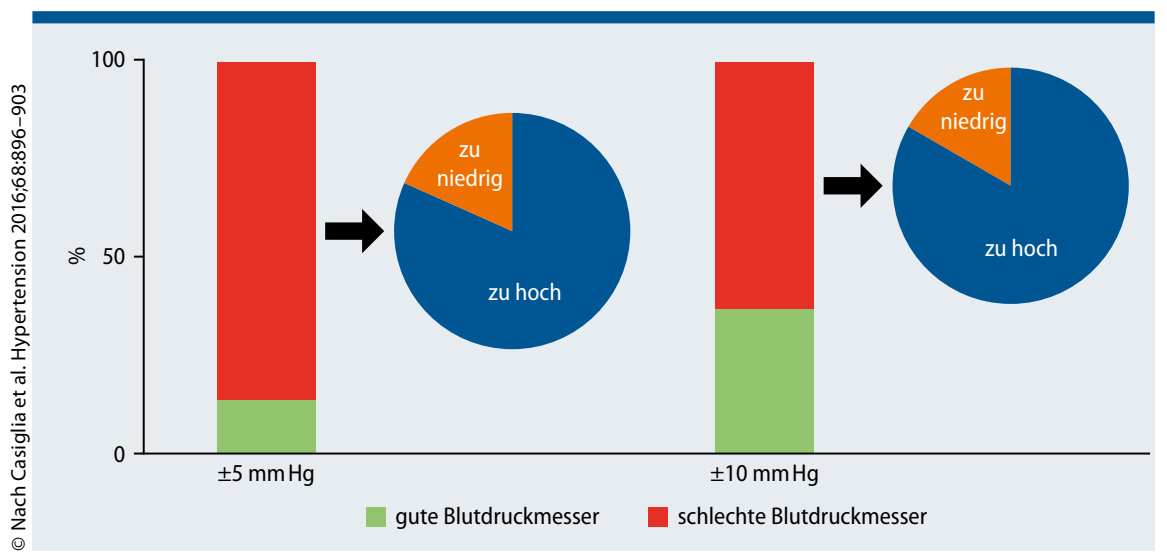

Anteil der Probanden, die innerhalb und außerhalb eines Messfehlers von \pm 5 bzw. 10 $\mathrm{mmHg}$ bei der Messung am Handgelenk lagen. Die Kreisdiagramme zeigen „schlechte Blutdruckmesser", die zu hoch oder zu niedrig gemessen haben. systolisch als auch diastolisch am Handgelenk höher als am Oberarm war (+5,6\% bzw. $+5,4 \%$, jeweils $\mathrm{p}<0,0001)$. 621 Probanden hatten einen häuslichen Messfehler von mindestens $\pm 5 \mathrm{mmHg}$, 455 von mindestens $\pm 10 \mathrm{mmHg}$ (schlechte Blutdruckmesser, siehe Abbildung).

Geringere kognitive Fähigkeiten beeinflussten sowohl die systolische als auch die diastolische Selbstmessung signifikant, eine größere Unterarmlänge nur die systolische Messung. Beide Faktoren dürften häufiger zu einer falschen Position des Handgelenks bei der Messung geführt haben.

Casiglia E et al. Poor Reliability of Wrist Blood Pressure Self-Measurement at Home: A Population-Based Study. Hypertension. 2016;68:896-903

\section{Kommentar}

Diese Untersuchung bestätigt den seit Langem bestehenden Eindruck, dass die Handgelenksmessung zwar die Akzeptanz der Selbstmessung erhöht haben dürfte, häufig aber zu wenig verlässlichen Ergebnissen führt. Denjenigen Patienten, denen wir ein exaktes Vorgehen bei der Selbstmessung am Handgelenk nicht zutrauen, sollten wir unbedingt zu der Messung am Oberarm raten.

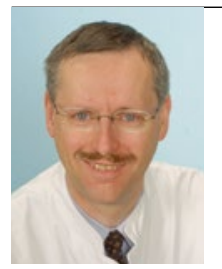

Prof. Dr. med. Walter Zidek Medizinische Klinik IV, Charité Universitätsmedizin Berlin 